\title{
Effects of acute left ventricular unloading on right ventricular function in normal and chronic right ventricular pressure-overloaded lambs
}

Boudewijn P. J. Leeuwenburgh, $\mathrm{MSc}^{\mathrm{a}, \mathrm{b}}$

Willem A. Helbing, MD ${ }^{\mathrm{a}, \mathrm{d}}$

Paul Steendijk, $\mathrm{PhD}^{\mathrm{b}}$

Paul H. Schoof, MD ${ }^{c}$

Jan Baan, $\mathrm{PhD}^{\mathrm{b}}$

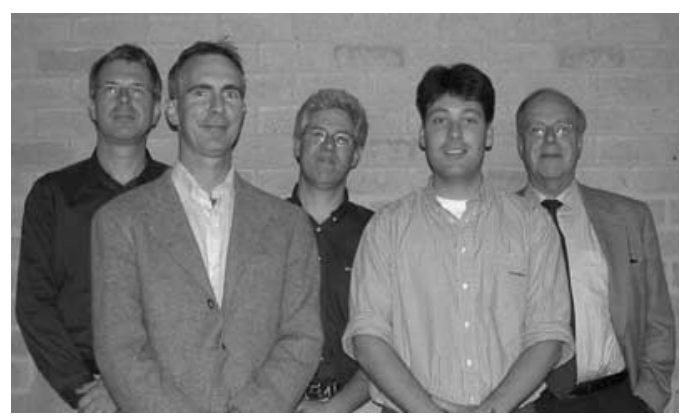

Helbing, Schoof, Steendijk, Leeuwenburgh, Baan (left to right)
Objective: Right ventricular pressure overload occurs in several types of (congenital) heart disease, as well as in pulmonary disease. Clinical outcome in some of these patient groups might in part be related to left ventricular loading conditions. The effects of left ventricular unloading on the function of the hypertrophic right ventricle have not been studied. We aimed to study the effects of left ventricular unloading on right ventricular hemodynamics and contractility in an animal model of chronic right ventricular pressure overload.

Methods: In lambs the pulmonary artery was chronically banded to increase right ventricular pressure to systemic levels. After 8 weeks, right ventricular contractility and hemodynamic function were assessed in these lambs, as well as in age-matched control animals, by using a combined pressure-conductance catheter in the right ventricle during baseline conditions and during complete bypass of the left ventricle.

Results: In both groups acute left ventricular unloading significantly decreased left ventricular pressure to low levels while aortic pressure was maintained. In the right ventricle of the control group, both end-systolic and end-diastolic volumes increased with left ventricular unloading $(P<.01)$ while end-systolic pressure was maintained. Cardiac output was unchanged despite decreased right ventricular contractility. In the banding group acute left ventricular unloading also decreased right ventricular contractility but increased cardiac output. During acute left ventricular unloading, diastolic stiffness was unchanged in the control group, whereas it was significantly decreased in the banding group.

Conclusions: Both in normal hearts and in hearts subject to chronic right ventricular pressure overload, acute left ventricular unloading decreases right ventricular contractility. Although no effects on cardiac output are encountered in normal hearts during left ventricular bypass, cardiac output is improved in right ventricular pressure-overloaded hearts, most likely related to improved right ventricular diastolic compliance.

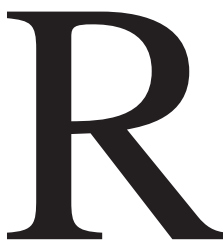

ight ventricular $(\mathrm{RV})$ pressure overload is common in several types of congenital and acquired heart disease. ${ }^{1}$ It may cause RV dysfunction or may result in residual abnormalities of RV function after relief of the abnormal load. ${ }^{1,2} \mathrm{RV}$ pressure overload occurs in otherwise normal hearts (eg, in pulmonary hypertension or isolated pulmonary stenosis), in hearts in which the right ventricle supports the systemic circulation and the left ventricle supports the (low-pressure) pulmonary
From the Departments of Pediatric CardiSurgery, ${ }^{\mathrm{c}}$ Leiden University Medical Cenrics (subdivision of Cardiology), ${ }^{\mathrm{d}}$ Erasmus Financial support by the Gisela Thier Founthe Leiden University Medical Center is gratefully acknowledged.

sions requested May 15, 2002, revisions received June 27, 2002; accepted for publication Aug 2, 2002

Address for reprints: J. Baan, PhD, Leiden Medical Center, Department of C-5-P, PO Box 9600, 2300 RC J.Baan@lumc.nl).

J Thorac Cardiovasc Surg 2003;125:481-90

Copyright () 2003 by The American Association for Thoracic Surgery

$0022-5223 / 2003 \$ 30.00+0$

doi: $10.1067 / \mathrm{mtc} .2003 .28$ 
circulation (eg, transposition of the great arteries), or in hearts in which the right ventricle is the only functional ventricle (eg, hypoplastic left-heart syndrome). Clinical outcome in these patient groups might be related to the different left ventricular (LV) loading conditions. Although several studies have been performed to investigate (systolic) ventricular interaction in the normal heart ${ }^{3}$ and a dependency of normal RV function on LV function has been documented,4-6 little is known about the effects of LV unloading on the function of the hypertrophic right ventricle. In addition to systolic ventricular interaction, the importance of diastolic ventricular interaction has also been recognized. ${ }^{7}$

In this study we used a combined pressure-conductance catheter to record pressure-volume (PV) loops in the right ventricle, which enabled us to study systolic and diastolic ventricular function at the same time.

The aim of our study was to investigate the effects of acute reductions in LV pressure established by means of total left-heart bypass (LHB) on the function of the hypertrophic right ventricle in lambs.

\section{Methods}

Ten lambs were enrolled in the study and treated according to the "Guide for the Care and Use of Laboratory Animals" published by the US National Institutes of Health (National Institutes of Health publication no. 85-23, revised 1996). The protocol was approved by the animal research committee of the Leiden University Medical Center. The first group consisted of 5 lambs that were 2 to 3 weeks old (mean body mass, $6.4 \pm 1.7 \mathrm{~kg}$ ) at the time of pulmonary artery banding (PAB). The animals were studied during a second operation (mean body mass at time of hemodynamic studies, $16.6 \pm 3.7 \mathrm{~kg}$ ) after a period of pulmonary artery (PA) constriction of at least 8 weeks (mean, $64 \pm 8$ days). A second group consisted of 5 control lambs (mean body mass, $20.4 \pm 3.0$ $\mathrm{kg}$ ) age matched with the PAB group.

\section{PAB Operation}

The procedure described below was developed in our laboratory and has been described previously. ${ }^{8}$ In brief, anesthesia was induced with propofol (4-6 mg/ $/ \mathrm{kg})$ and maintained with a mixture of $0.5 \%$ to $1.5 \%$ isoflurane and oxygen and continuous intravenous infusion of propofol $(6-18 \mathrm{mg} / \mathrm{kg} / \mathrm{h})$. The lambs were artificially ventilated with a volume-controlled respirator (Servo 900B; Siemens-Elema, AB, Solna, Sweden). Analgesia was provided with a combination of ramifenazone and fenylbutazone (Tomanol; 0.03 $\mathrm{mL} / \mathrm{kg}$ administered intravenously).

The chest was opened, and the heart was exposed in a pericardial cradle. A bidirectionally adjustable hydraulic occluder (noninflated lumen diameter of $12 \mathrm{~mm}$ ) attached to a subcutaneous reservoir was placed loosely around the PA (UNO, Zevenaar, The Netherlands). In addition, 2 pressure lines $(2.1 \mathrm{~mm}$ OD $\times 1.0 \mathrm{~mm}$ ID) attached to subcutaneous reservoirs $(0.25 \mathrm{~mL}$, UNO) were inserted into the carotid artery and into the right ventricle through a minor stab wound in the free wall. The pericardium was approx- imated for two thirds to support the heart during the period of RV pressure overload, and the thorax was closed in layers. Complete pericardial closure could not be achieved because it would have interfered with the position of the PA occluder and would have produced an unphysiologically high degree of constriction. ${ }^{9}$ After 1 week of recovery from the operation, the cuff was inflated by means of stepwise injection of hypertonic saline into the reservoir over a period of 2 weeks until the right ventricle faced a pressure equal to the systemic (aortic) pressure. During the next 8-week period, RV peak systolic pressure was kept at the level of peak aortic systolic pressure through biweekly monitoring of RV and aortic pressures by using the subcutaneous reservoirs, followed by PA cuff adjustments, if necessary.

\section{Data Acquisition and Experimental Protocol}

After at least 8 weeks of chronic RV pressure overload, anesthesia was initiated with sodium thiopental $(10 \mathrm{mg} / \mathrm{kg}$ administered intravenously) in 5 PAB lambs and 5 age-matched control animals. Ventilation and monitoring of the animals during the experiment were identical to that during the $\mathrm{PAB}$ procedure. Before chest opening, pancuronium bromide $(0.1 \mathrm{mg} / \mathrm{kg}$; a muscle relaxant) was administered.

A midsternal (re)thoracotomy was performed. The pericardium was widely opened, all pericardial adhesions resulting from the initial operation were completely removed from around the heart, and the heart was (re)exposed in a pericardial cradle. A $5 \mathrm{~F}$ pigtailed combined pressure-conductance catheter (Millar Instruments, Houston, Tex) was positioned in the right ventricle through a minor stab wound just below the pulmonary valve and positioned toward the apex for continuous and simultaneous measurement of pressure and volume. ${ }^{10}$ Another pressure catheter was inserted into the left ventricle (Millar Instruments). For calibration of the conductance catheter, a 7F Swan-Ganz catheter was placed in the PA. ${ }^{11} \mathrm{PV}$ relationships were obtained by means of preload reduction with a string around the inferior vena cava.

LV pressure unloading was performed by means of total LHB with a centrifugal pump (Sarns Delphin system; 3M, Ann Arbor, Mich). A $16 \mathrm{~F}$ arterial return cannula was inserted into the ascending aorta, and a venous withdrawal cannula (32F) was inserted into the LV apex. The withdrawal cannula emptied into a custom-made temperature-controlled glass reservoir that was connected to the centrifugal pump head. Figure 1 shows a schematic drawing of the experimental preparation used for LV unloading.

The conductance catheter in the right ventricle was connected to a Leycom Sigma-5 DF signal processor (CD Leycom, Zoetermeer, The Netherlands) and calibrated as previously described ${ }^{11}$ : RV parallel conductance was determined by using hypertonic saline injections, ${ }^{12}$ and the slope factor $\alpha$ was determined by means of thermodilution.

After instrumentation, a 10-minute stabilization period was allowed before baseline measurements were obtained. Data acquisition was performed as described elsewhere. ${ }^{11}$ After completion of baseline measurements, LV unloading was initiated by means of total LHB. Pump speed (and thus reservoir level) was adjusted to achieve an LV end-systolic pressure $\left(\mathrm{P}_{\mathrm{ES}}\right)$ of less than $30 \mathrm{~mm} \mathrm{Hg}$ and a mean aortic pressure of greater than $60 \mathrm{~mm} \mathrm{Hg}$. The mean aortic pressure signal was flat, indicating a closed aortic valve throughout systole (Figure 2). Once steady-state conditions had 


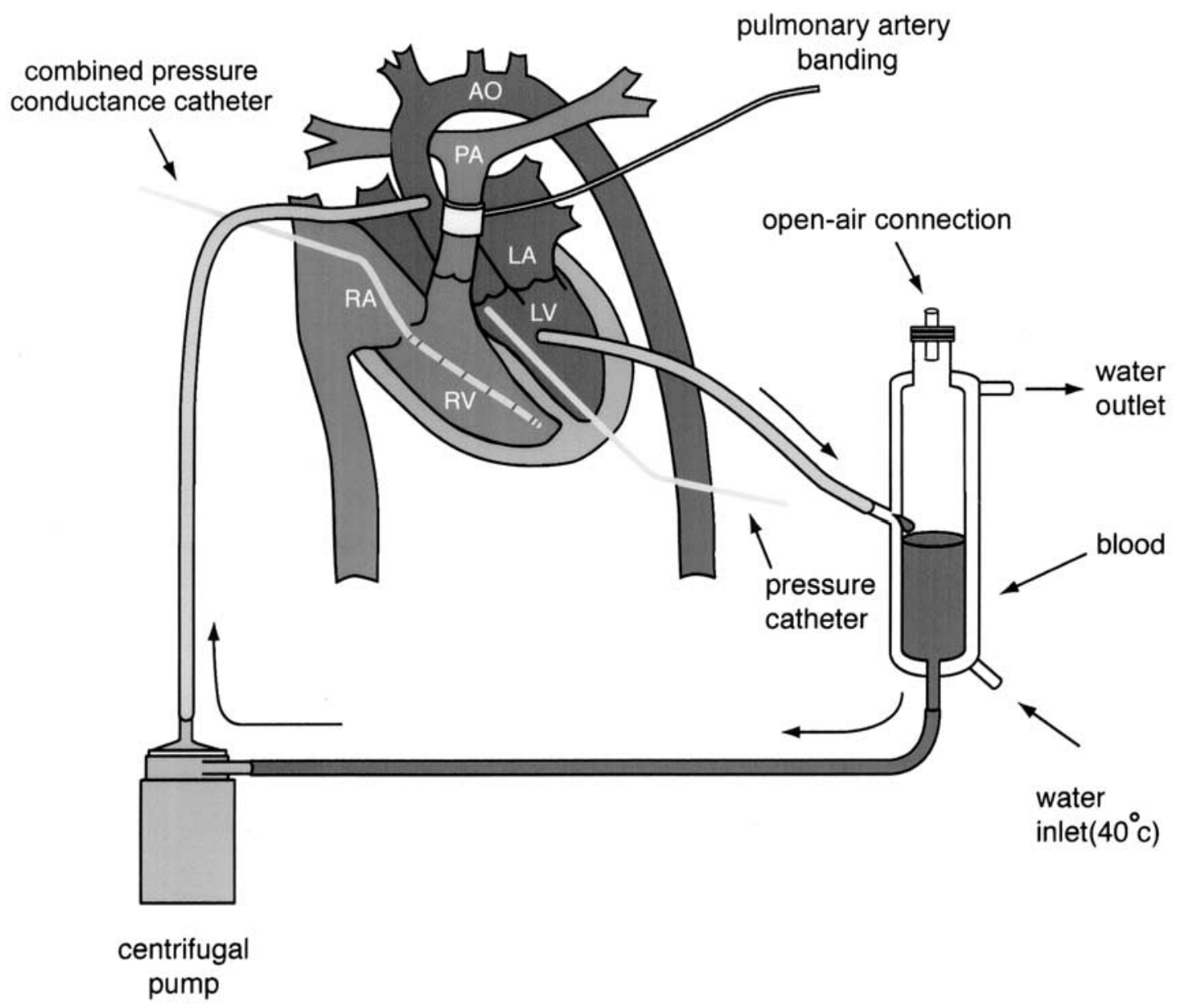

Figure 1. Schematic representation of the instrumentation for total LHB. Blood is removed from the LV apex and collected in a temperature-controlled glass reservoir. Through a centrifugal pump, blood is propelled from the reservoir back to the ascending aorta. Before LV bypass, the reservoir was filled with donor blood from another sheep to a fixed reference level. Pump speed was adjusted to maintain this reference level in the reservoir. Note that because of the interpositioning of the open reservoir, blood was removed passively from the left ventricle both because of a height difference between the mid-left ventricle and the reservoir and by the pumping action of the left ventricle itself (no active $L V$ suction by the bypass pump). AO, Aorta; $P A$, pulmonary artery; $L A$, left atrium; $R A$, right atrium; $L V$, left ventricle; $R V$, right ventricle.

been achieved, pump speed was not changed. After proper calibration, data were recorded during transient vena caval occlusion to assess RV contractility. ${ }^{11,13-15}$ At the end of the experiment, the animals were killed by means of injection of $20 \mathrm{~mL}$ of $\mathrm{KCl}$ after achievement of adequate anesthesia.

\section{Data Analysis}

For both conditions, steady-state data were recorded for at least 10 seconds to determine the hemodynamic parameters listed in Table 1. Stroke work was calculated as the PV loop area for each beat. Data recorded during the vena caval occlusions were used to construct the following PV relationships: the end-systolic PV relationship (ESPVR), ${ }^{16}$ the $\mathrm{dP} / \mathrm{dt}_{\max }$ versus end-diastolic volume $\left(\mathrm{dP} / \mathrm{dt}_{\max }-\mathrm{V}_{\mathrm{ED}}\right)$ relationship, ${ }^{17}$ and preload recruitable stroke work (stroke work vs $\mathrm{V}_{\mathrm{ED}}$ relationship or PRSW). ${ }^{18}$ The volume inter- cept of the ESPVR and the slopes of the above 3 relationships were used as relatively load-independent indices of RV contractility, ${ }^{11,13,14,19,20}$ as previously described for the left ventricle. ${ }^{16-18,21}$ Because PAB increased RV pressure considerably, the pressure level of the volume intercept of the ESPVR was chosen at $15 \mathrm{~mm}$ $\mathrm{Hg}\left(\mathrm{V}_{15}\right)$ in the control group and at $55 \mathrm{~mm} \mathrm{Hg}\left(\mathrm{V}_{55}\right)$ in the banding group. Therefore, direct comparison of the volume intercepts between the 2 groups (ie, $\mathrm{V}_{15}$ and $\mathrm{V}_{55}$ ) is not meaningful. The volume intercepts, however, were used for the evaluation of LV unloading within each group, as previously described. ${ }^{8}$ The diastolic stiffness constant (b) was derived from the end-diastolic PV relationship (EDPVR), which was obtained by fitting enddiastolic pressure $\left(\mathrm{P}_{\mathrm{ED}}\right)$ and volume data points to a monoexponential equation, as described elsewhere. ${ }^{22}$ 

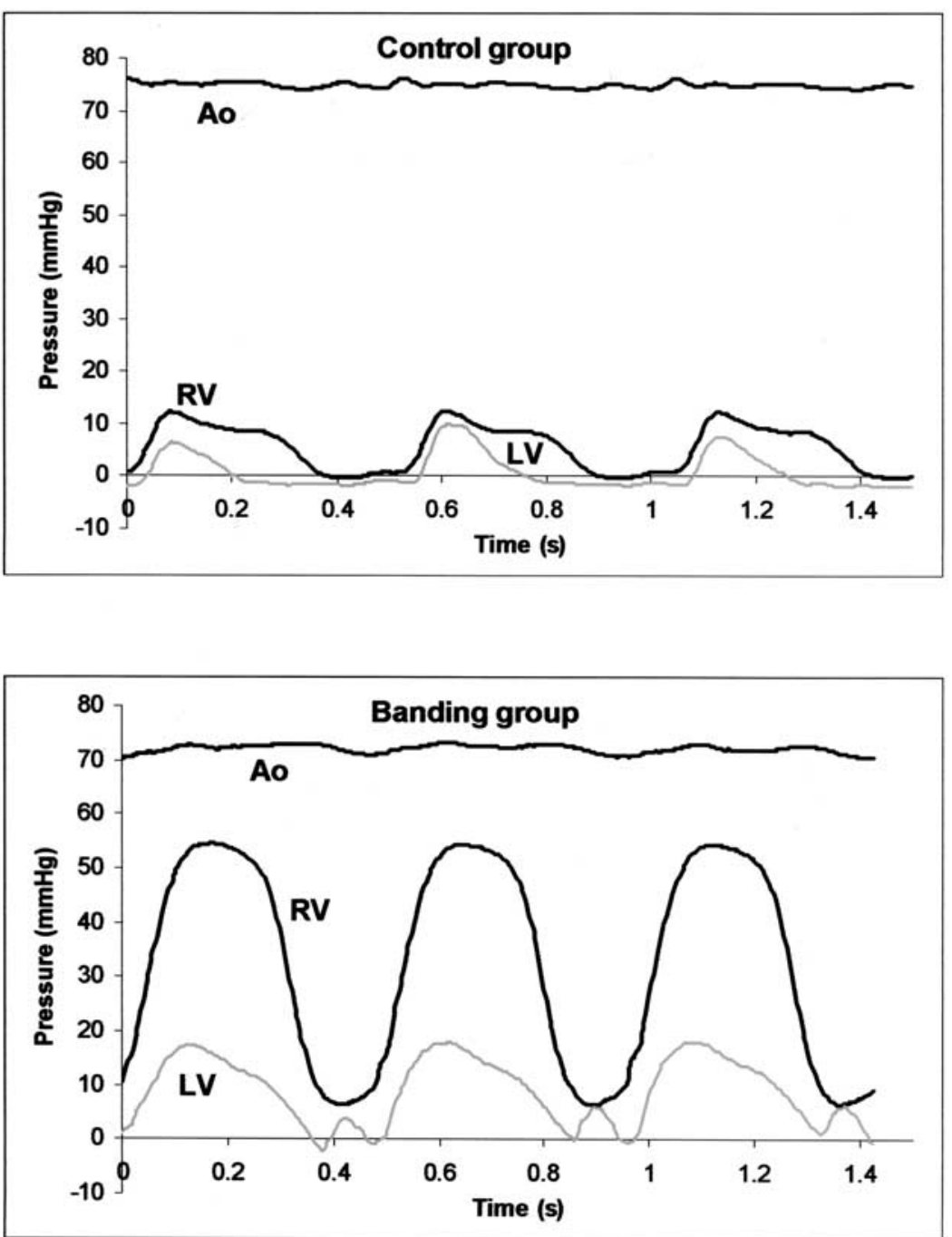

Figure 2. Typical example of pressure tracings during LV bypass in the control (upper panel) and banding (lower panel) groups. The lower black line represents the RV pressure signal (RV), the gray line represents the LV pressure signal (LV), and the black line at the top represents aortic pressure (Ao). Note that the aortic pressure signal is flat, indicating a closed aortic valve during LV bypass. Although the diastolic pressure interval during LHB in the banding group suggests a positive pressure peak, calculated average LV pressures in these diastolic intervals in both groups indicate that this is an inconsistent finding (control group, $-1.7 \pm 1.1 \mathrm{~mm} \mathrm{Hg}$; banding group, $-2.2 \pm$ $4.7 \mathrm{~mm} \mathrm{Hg}$ ).

\section{Statistical Analysis}

The effects of LV unloading on hemodynamic parameters were analyzed for each group separately by using a multiple linear regression implementation of repeated-measures analysis of variance. ${ }^{23}$ Between-group differences and the effect of LV unloading were tested by using the unpaired Student $t$ test. Data are presented as means $\pm \mathrm{SD}$.

\section{Results}

Table 1 shows the average RV hemodynamic data for both groups during baseline conditions and during acute LV unloading. Indices of RV contractility derived from PV loop analysis are summarized in Table 2. Figure 3 shows typical examples of PV loops in the right ventricles of both groups. In Figure 4 average schematic RV PV loops are shown, summarizing the effects of the acute decrease in LV pressure on RV function.

Effects of LV Pressure Unloading in Normal Hearts In the control group total LHB decreased average LV $\mathrm{P}_{\mathrm{ES}}$ from $77 \pm 32$ to $29 \pm 38 \mathrm{~mm} \mathrm{Hg}(P<.01)$, whereas mean 
TABLE 1. RV hemodynamic parameters

\begin{tabular}{|c|c|c|c|c|c|c|c|c|}
\hline & \multicolumn{3}{|c|}{ Control group } & \multicolumn{3}{|c|}{ Banding group } & \multirow{2}{*}{$\begin{array}{c}\text { PAB effect, } \\
P \text { value }\end{array}$} & \multirow{2}{*}{$\begin{array}{c}\text { Bypass effect, } \\
\boldsymbol{P} \text { value }\end{array}$} \\
\hline & Baseline & LV bypass & $P$ value & Baseline & LV bypass & $P$ value & & \\
\hline HR (beats/min) & $130 \pm 18$ & $122 \pm 24$ & .22 & $128 \pm 22$ & $137 \pm 33$ & .14 & .60 & .19 \\
\hline $\mathrm{CO}(\mathrm{mL} / \mathrm{min})$ & $2369 \pm 473$ & $2383 \pm 989$ & .55 & $1612 \pm 432$ & $2130 \pm 1226$ & .03 & .02 & .34 \\
\hline SV (mL) & $18.2 \pm 2.2$ & $19.1 \pm 4.6$ & .21 & $12.5 \pm 1.5$ & $14.8 \pm 5.5$ & .02 & $<.01$ & .54 \\
\hline $\mathrm{V}_{\mathrm{ES}}(\mathrm{mL})$ & $13.6 \pm 1.2$ & $16.4 \pm 4.5$ & .03 & $19.3 \pm 9.2$ & $26.5 \pm 23.3$ & .01 & .08 & .80 \\
\hline$V_{E D}(m L)$ & $30.6 \pm 2.7$ & $33.8 \pm 7.9$ & .05 & $29.2 \pm 8.1$ & $35.9 \pm 20.6$ & $<.01$ & .98 & .78 \\
\hline $\mathrm{EF}(\%)$ & $59 \pm 3$ & $57 \pm 9$ & .50 & $43 \pm 10$ & $44 \pm 10$ & .57 & $<.01$ & .16 \\
\hline$P_{E S}(m m ~ H g)$ & $15 \pm 2$ & $13 \pm 3$ & .09 & $52 \pm 16$ & $55 \pm 23$ & .21 & $<.01$ & .09 \\
\hline$P_{E D}(m m ~ H g)$ & $2 \pm 2$ & $3 \pm 1$ & .12 & $7 \pm 3$ & $7 \pm 4$ & .47 & $<.01$ & .82 \\
\hline $\mathrm{SW}(\mathrm{mm} \mathrm{Hg} \cdot \mathrm{mL})$ & $396 \pm 227$ & $383 \pm 249$ & .78 & $557 \pm 284$ & $631 \pm 347$ & .01 & .14 & .05 \\
\hline $\mathrm{dP} / \mathrm{dt}_{\mathrm{Max}}(\mathrm{mm} \mathrm{Hg} / \mathrm{s})$ & $745 \pm 508$ & $594 \pm 372$ & .03 & $795 \pm 246$ & $976 \pm 468$ & .07 & .40 & .03 \\
\hline $\mathrm{b}\left(\mathrm{mL}^{-1}\right)$ & $0.16 \pm 0.10$ & $0.21 \pm 0.07$ & .13 & $0.30 \pm 0.09$ & $0.20 \pm 0.07$ & .05 & .04 & .04 \\
\hline
\end{tabular}

$P$ values indicate statistical significance for the difference between baseline conditions and LV bypass in each group. $P A B$ effect indicates $P$ values for the difference in baseline conditions between the control and banding groups, and bypass effect indicates $P$ values for differences of LHB between groups. $H R$, Heart rate; $C O$, cardiac output; $S V$, stroke volume; $E F$, ejection fraction; $d P / d t_{M a x}$ maximum of the first derivative of pressure versus time; $b$, diastolic

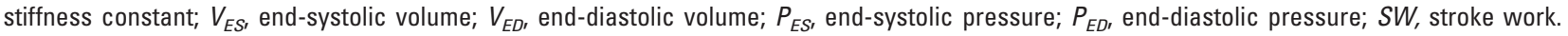

TABLE 2. Indices of RV contractility

\begin{tabular}{|c|c|c|c|c|c|c|}
\hline & \multicolumn{3}{|c|}{ Control } & \multicolumn{3}{|c|}{ Banding } \\
\hline & Baseline & Low afterload & $P$ value & Baseline & Low afterload & $P$ value \\
\hline $\mathrm{E}_{\mathrm{ES}}(\mathrm{mm} \mathrm{Hg} / \mathrm{mL})$ & $1.2 \pm 0.5$ & $1.0 \pm 0.4$ & .64 & $5.2 \pm 2.8$ & $3.0 \pm 1.9$ & $<.01$ \\
\hline $\mathrm{V}_{15}(\mathrm{~mL})$ & $15.4 \pm 5.6$ & $16.2 \pm 5.0$ & .01 & - & - & - \\
\hline$V_{55}(\mathrm{~mL})$ & - & - & - & $20.0 \pm 8.9$ & $27.5 \pm 23.5$ & .04 \\
\hline $\mathrm{S}-\mathrm{dP} / \mathrm{dt}\left(\mathrm{mm} \mathrm{Hg} \cdot \mathrm{s}^{-1} \cdot \mathrm{mL}^{-1}\right)$ & $16.2 \pm 14.0$ & $7.2 \pm 6.8$ & .07 & $29.5 \pm 15.2$ & $24.0 \pm 6.2$ & .55 \\
\hline S-PRSW (mm Hg) & $16.3 \pm 8.3$ & $14.4 \pm 5.3$ & .01 & $39.0 \pm 21.8$ & $27.1 \pm 11.5$ & .14 \\
\hline
\end{tabular}

$P$ values indicate statistical significance for the difference between baseline conditions and LV bypass in each group.

$E_{E S}$, End-systolic elastance; $V_{15 / 55}$ intercept of the ESPVR with the volume axis at an end-systolic pressure of 15 (control group) or $55 \mathrm{~mm} \mathrm{Hg}$ (banding group); $S-d P / d t$, slope of the $\mathrm{dP} / \mathrm{dt}_{\mathrm{Max}}-\mathrm{V}_{\mathrm{ED}}$ relationship; $S-P R S W$, slope of the preload recruitable stroke work relationship.

aortic pressure averaged $63 \pm 20 \mathrm{~mm} \mathrm{Hg}$. Cardiac output and RV stroke work did not differ from baseline levels (Table 1). However, both end-systolic volume $\left(\mathrm{V}_{\mathrm{ES}}\right)$ and $\mathrm{V}_{\mathrm{ED}}$ of the $\mathrm{RV}$ increased, resulting in a small but significant rightward displacement of the entire RV PV loop (Figure 4). RV $\mathrm{P}_{\mathrm{ES}}$ did not change ( $15 \pm 2$ to $\left.13 \pm 3 \mathrm{~mm} \mathrm{Hg}, P=.09\right)$. The slope of the RV ESPVR was unchanged, but its volume intercept $\left(\mathrm{V}_{15}\right)$ increased from $15.4 \pm 5.6$ to $16.2 \pm 5.0 \mathrm{~mL}$ $(P<.01)$, indicating decreased RV contractility. Two other indices of contractility, the slopes of the PRSW- and $\mathrm{dP} /$ $\mathrm{dt}_{\mathrm{Max}}-\mathrm{V}_{\mathrm{ED}}$ relationships, were also decreased, although the latter was only marginally significant $(P=.07$, Table 2$)$. These findings indicate reduced RV contractility during LHB in the control group, whereas cardiac output is maintained at the expense of increased $\mathrm{V}_{\mathrm{ED}}$. RV diastolic compliance did not change during acute reductions in LV pressure, as indicated by the RV diastolic stiffness constant $\mathrm{b}$ $\left(0.16 \pm 0.10\right.$ to $0.21 \pm 0.07 \mathrm{~mL}^{-1}, P=$ not significant $)$.

\section{Effects of LV Pressure Unloading in RV Pressure- overloaded Hearts}

Chronic RV pressure overload resulted in a significantly increased $\mathrm{RV} / \mathrm{LV}$ wall-thickness ratio from $0.43 \pm 0.04$ to
$0.94 \pm 0.15(P<.01)$, indicating substantial RV hypertrophy. In the banding group, LHB decreased LV $\mathrm{P}_{\mathrm{ES}}$ from $68 \pm 16$ to $28 \pm 21 \mathrm{~mm} \mathrm{Hg}(P<.01)$, whereas mean aortic pressure averaged $71 \pm 34 \mathrm{~mm} \mathrm{Hg}$, which is not significantly different from the mean aortic pressure in the control group. $\mathrm{RV} \mathrm{P}_{\mathrm{ES}}$ did not change significantly $(52 \pm 16$ to $55 \pm 23 \mathrm{~mm} \mathrm{Hg}, P=.21)$. Cardiac output increased significantly, which was due to an increase in stroke volume. Thus whereas in the control group LV unloading did not affect cardiac output, in the banding group cardiac output was improved by LV unloading. Again, LHB increased both $\mathrm{V}_{\mathrm{ES}}$ and $\mathrm{V}_{\mathrm{ED}}$ in the right ventricle (both $P<$ .01 , Table 1), resulting in a significant rightward shift of the entire PV loop (Figure 4). RV systolic function was improved, as indicated by increased stroke work (from $557 \pm$ 284 to $631 \pm 347 \mathrm{~mm} \mathrm{Hg} \cdot \mathrm{mL}, P<.01)$. The increase in stroke volume and stroke work are both related to the enhanced preload reflected by $\mathrm{V}_{\mathrm{ED}}$. In contrast, $\mathrm{RV}$ contractility was significantly decreased, as indicated by both a decreased slope (from $5.2 \pm 2.8$ to $3.0 \pm 1.9 \mathrm{~mm} \mathrm{Hg} / \mathrm{mL}$, $P<.01)$ and an increased volume intercept $\left(\mathrm{V}_{55}\right)$ of the ESPVR (from $20.0 \pm 8.9$ to $27.5 \pm 23.5 \mathrm{~mL}, P<.05$; 

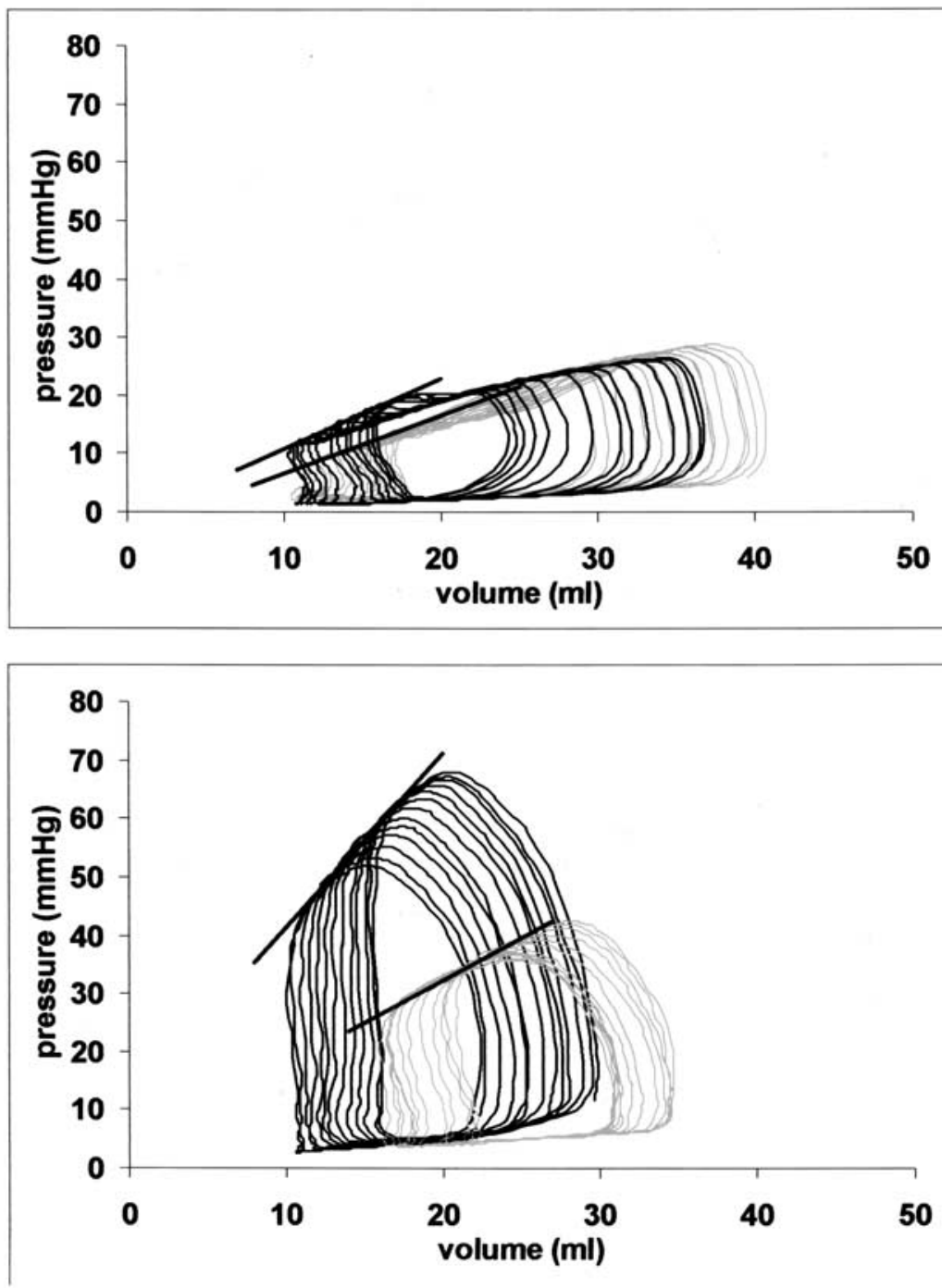

Figure 3. Typical recordings of RV PV loops during a vena caval occlusion in the control (top panel) and banding (lower panel) groups. Black loops indicate the baseline condition, whereas gray loops were recorded during acute decreases in LV pressure. ESPVRs are represented by straight black lines.

Table 2 and Figure 4). The 2 other indices of contractility showed a tendency to decrease but failed to reach statistical significance (Table 2). RV diastolic stiffness in the baseline condition was increased compared with the baseline condition in the control group, as evidenced by the increased value of $\mathrm{b}$ (from $0.16 \pm 0.10$ to $0.30 \pm 0.09 \mathrm{~mL}^{-1}, P<$ .05). During acute reductions in LV pressure, however, RV diastolic stiffness in the banding group was significantly decreased to the near-baseline levels found in the control group $\left(0.30 \pm 0.09\right.$ to $0.20 \pm 0.07 \mathrm{~mL}^{-1}, P<.05$; Table 1$)$.

Significant changes between both groups in terms of a different effect of LV bypass were found for stroke work, $\mathrm{dP} / \mathrm{dt}_{\mathrm{Max}}$, and the chamber stiffness constant b. LHB did not have a significantly different effect on the other parameters listed in Table 1.

\section{Discussion}

The results of this study demonstrate that in the normal heart cardiac output is not affected by acute reductions in LV pressure. After chronic RV pressure overload, resulting in substantial RV hypertrophy, baseline cardiac output is significantly decreased (Table 1). However, during acute reductions in LV pressure in this group, cardiac output is improved, resulting from a significant increase in stroke volume. The observed marginal but nonsignificant increase in heart rate might reflect some autonomic reflex activity 

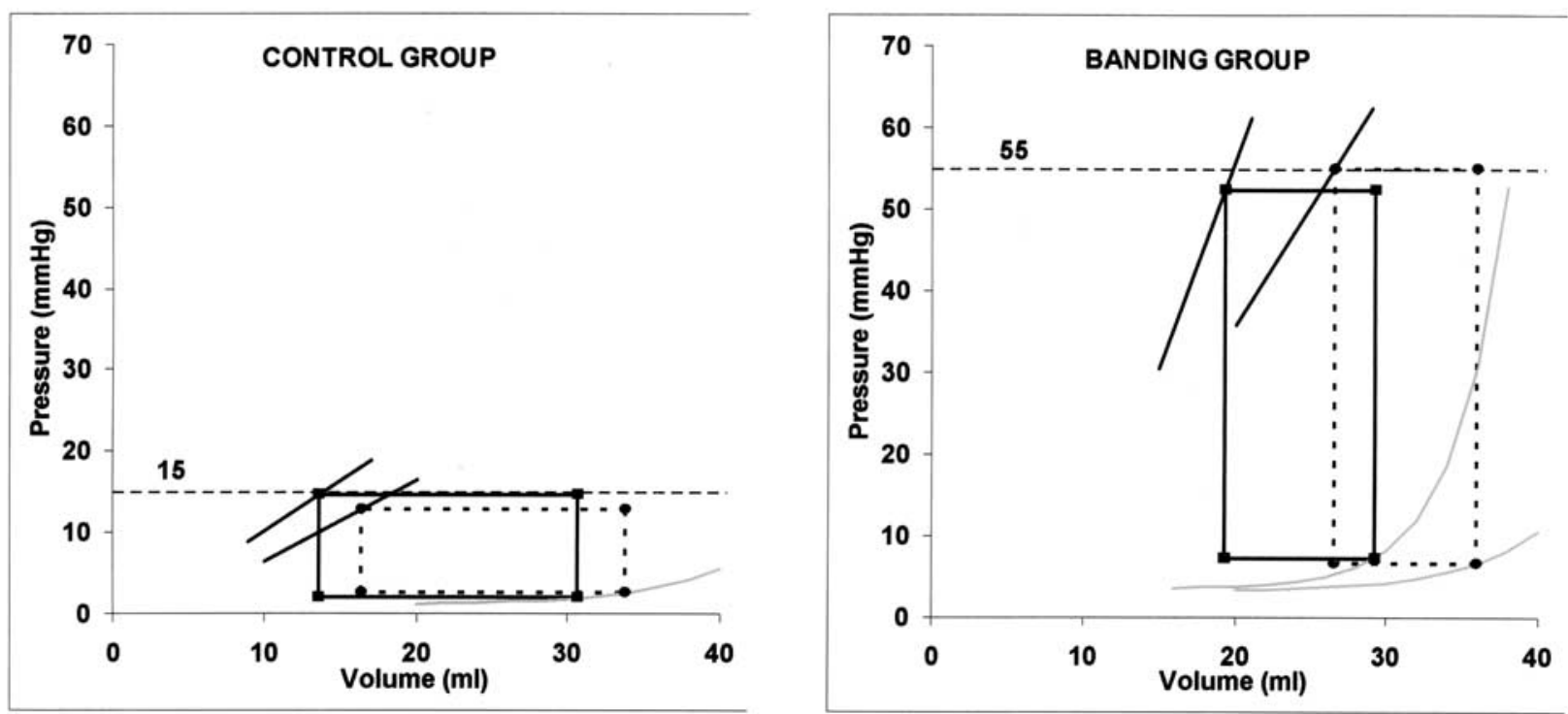

Figure 4. Schematic average RV PV loops and corresponding average ESPVRs (solid black lines) and EDPVRs (solid gray lines) showing the effects of LV bypass in the control group (left panel) and in the banding group (right panel). Solid loops represent the baseline condition, whereas dotted loops represent the LV bypass condition. The thin horizontal lines indicate the intercept with the ESPVR at an end-systolic pressure of 15 and $55 \mathrm{~mm} \mathbf{~ H g}$, respectively. Note that in the control group the average PV loop shifts rightward along the same EDPVR during LV unloading, whereas in the banding group the average PV loop shifts to the right along a different EDPVR.

because aortic pressure is changed from a pulsatile to a flat flow pattern during LV bypass (Figure 3). In both groups, RV contractility was found to be decreased as indicated by a rightward shift of the entire PV loop and with a concomitant increase of the volume intercept of the ESPVR, by a decrease of the slope of the ESPVR ( $\left.\mathrm{E}_{\mathrm{ES}}\right)$, or both.

Several studies evaluated the effect of LV pressure unloading on RV function in the normal heart, but the results of these experiments are controversial. In response to $\mathrm{LV}$ bypass, global RV function has been found to be improved, ${ }^{15,24}$ decreased, ${ }^{25,26}$ or unchanged. ${ }^{3,6,27}$ The effects of LV bypass on the function of the hypertrophied right ventricle, however, have not been studied.

An unexpected finding of our study is the significant increase in cardiac output during LV unloading in the banding group. The chronic RV pressure overload itself has led to a significant decrease in cardiac output compared with that seen in the control group ( $2369 \pm 473$ vs $1612 \pm 432$ $\mathrm{mL} / \mathrm{min}$, respectively; $P<.01$ ), as previously described. ${ }^{8}$ This substantial chronic decrease in cardiac output in the banding group was reversed during LV bypass because cardiac output proved to be acutely increased and returned to near-baseline levels, as found in the control group (Table 1). Although these findings suggest that in the acute situation LV bypass will improve cardiac output in the banding group, it might have detrimental effects on RV function in the long run because RV volume is increased, which is an early hallmark of remodeling.
Close inspection of the diastolic portion of the average PV loops might provide an explanation for the improved cardiac output in the banding group (Figure 4, right panel). The considerable rightward shift of the PV loop after LV unloading and the numbers in Table 1 indicate that $\mathrm{P}_{\mathrm{ED}}$ remains unchanged despite the substantial increase in $\mathrm{V}_{\mathrm{ED}}$, which suggests an improvement in RV diastolic compliance. This is further substantiated by the significantly different effect of LV bypass on the diastolic stiffness constant between the groups (bypass effect, Table 1). Analysis of the EDPVR in both groups indicated that although the resting value of the diastolic stiffness constant $b$ in the hypertrophic right ventricle is significantly higher compared with that found in the normal right ventricle $(0.16 \pm 0.10$ to $0.30 \pm$ $0.09 \mathrm{~mL}^{-1}, P<.05$ ), acute $\mathrm{LV}$ unloading decreases the diastolic stiffness constant in the hypertrophic right ventricle by approximately $33 \%$ (from $0.30 \pm 0.09$ to $0.20 \pm 0.07$ $\left.\mathrm{mL}^{-1}, P<.05\right)$. In the absence of such a beneficial change, $\mathrm{P}_{\mathrm{ED}}$ would have increased along with $\mathrm{V}_{\mathrm{ED}}$ on the exponential EDPVR. Again, this is shown in Figure 4. The average PV loop in the control group (Figure 4, left panel) shifts to the right along the same EDPVR during acute LV unloading, whereas the average PV loop in the banding group (Figure 4, right panel) shifts to the right along a different EDPVR, which is displaced to the right and downward compared with that in the EDPVR in baseline conditions. This enhanced compliance most likely represents the mechanism by which RV filling is facilitated, thus increasing 
cardiac output during LV bypass. Similar observations have been reported by Fukamachi and coworkers, ${ }^{24}$ who found, in open-chest dogs, that LV bypass in the normal heart had a beneficial influence on RV performance through an increase in chamber compliance and a decrease in pulmonary arterial input resistance. Although in our study RV $\mathrm{P}_{\mathrm{ES}}$ did not change, it is obvious that this fact, together with the increased cardiac output, also implies reduced pulmonary vascular resistance (reflected roughly by $\mathrm{P}_{\mathrm{ES}} /$ cardiac output).

In normal hearts, LV contraction is known to contribute for $20 \%$ to $40 \%$ to RV pressure development and output during a normal cardiac cycle. ${ }^{28}$ During LV unloading, this LV-to-RV contribution is lacking. ${ }^{4}$ We speculate that the observed reduction in RV contractility on LV unloading in the control group is a direct result of the lacking LV pressure development, which might decrease septal stiffness. Despite the reduction in RV contractility, RV end-systolic pressure and stroke volume were maintained, which is most likely related to a septal shift. Septal shifting (besides the pericardium) represents one of the 2 mechanisms by which both ventricles are able to interact. In addition to this direct mechanical interaction, series interaction through the systemic and pulmonary circulations is a second mechanism that influences ventricular interaction. Previous studies in which either ultrasonic crystals or echocardiography were used, showed that a reduction of the septal pressure gradient during LV bypass is accompanied by a leftward septal shift. ${ }^{3,29-31}$ From these studies, it can be inferred that the pressure gradient across the interventricular septum is an important determinant of its position. In the control group of the current study, both $\mathrm{V}_{\mathrm{ES}}$ and $\mathrm{V}_{\mathrm{ED}}$ were increased approximately equally during LV bypass, whereas, consequently, stroke volume was unchanged (Table 1). Together with the finding of a $74 \%$ decrease in the transseptal pressure gradient (from $62 \pm 33$ to $16 \pm 40 \mathrm{~mm} \mathrm{Hg}, P<.01$ ), we conclude that the RV volume increase during LV bypass is most likely the result of a leftward septal shift, indicating (slight) RV geometric remodeling. In contrast, if no septal shift would have occurred and the rightward displacement of the RV end-diastolic volume point in Figure 4 was merely the result of an increase in preload (venous return), we would have expected an increase in ejection fraction after length-dependent activation (Frank-Starling mechanism). However, this is clearly not the case (Table 1). In the banding group, RV contractility was also found to be decreased during LV bypass, whereas cardiac output was increased. Similar to the effects in the control group, the pressure gradient was significantly reduced by LV bypass (from $15 \pm 22$ to $-27 \pm 23 \mathrm{~mm} \mathrm{Hg}, P<.05$ ). However, compared with values in the control group, the pressure gradient in the baseline condition was already low in the chronically pressure-overloaded heart. LV bypass not only lowered the pressure gradient further but even reversed it to the RV-to-LV direction in the banding group. Without doubt, this is accompanied, to a certain degree, by geometric remodeling. In a recent study it was found that by constricting the PA, circumferential compressive stresses develop in the septum that might impede septal blood flow and thus might induce septal ischemia. ${ }^{32}$ Although we cannot exclude the occurrence of septal ischemia after abnormal septal curvatures (remodeling), aortic pressure was maintained at greater than the critical level for coronary autoregulation at all times during LV bypass. Global ventricular ischemia as an explanation for the decreased RV contractility in our study is therefore not likely.

As for the series interaction, the bypass system that is normally used in the operating room might influence cardiac output because it is a closed fluid-filled system driven by a pump. Depending on the circulatory status of the patient, this might improve or worsen the cardiovascular condition. In contrast, in this study we used a custom-made bypass system consisting of a reservoir with an open-air connection (Figure 1). The advantage of this setup is that it enabled the left ventricle to eject passively into the reservoir without being influenced by the pump itself (ie, the suction effects of the pump did not affect the left ventricle). Because pump speed was adjusted to maintain a constant blood level in the reservoir before measurements were started, and normal interaction between the left ventricle and right ventricle, which influences stroke volume in the normal heart, is disabled by the bypass system, this allowed the right ventricle to regulate and modify cardiac output on its own accordingly.

In the clinical setting, chronic RV hypertrophy might be associated with a wide range of LV pressures (from decreased to increased) or even with absence of the left ventricle. LV function and volume might also vary widely. It has been suggested that with chronic RV hypertrophy, the presence of a left ventricle with normal pressures contributes to the preservation of RV function. ${ }^{33,34}$ In addition, Sano and colleages ${ }^{35}$ studied patients with congenitally corrected transposition of the great arteries and found deterioration of $\mathrm{RV}$ function after relief of a PA stenosis, which resulted in decreased LV pressure. This is not directly supported by our results. Although significant differences between the chronic situations in patients and our experiment are evident, our results seem to suggest that diastolic interplay between the hypertrophic right ventricle and the left ventricle is an important aspect of the performance of the hypertrophic right ventricle. This should be taken into account when interventions are planned that will alter biventricular compliance.

\section{Study Limitations}

After careful consideration, we decided not to perform a sham operation in the control group. It has been shown 
previously in lambs that cardiovascular function had recovered completely after thoracotomy with pericardiotomy as soon as 3 days postoperatively compared with that seen in healthy control animals. ${ }^{36}$ In addition, it is highly unlikely that the insertion of a small pressure line in the right ventricle affects cardiovascular function after a period of 8 weeks. During the second operation, all pericardial adhesions were completely removed around the heart, which was re-exposed in a pericardial cradle. It does not appear to be justified to subject healthy animals to a thoracotomy when the effects of the operation on cardiac function are not expected until after 8 weeks.

\section{Conclusions}

Both in normal hearts and in hearts subjected to chronic RV pressure overload at the systemic level, complete bypass of the left ventricle results in decreased RV contractility, which might be explained by changes in septal geometry (remodeling) and function. Although RV volume is increased in normal hearts during LV unloading, RV stroke volume remains unaffected. In contrast, in the chronically pressure-overloaded right ventricle, cardiac output is improved during acute LV unloading, and RV volume is increased. This might be explained by an increase in diastolic RV compliance.

We thank the biotechnicians of the Large Animal Laboratory of the Leiden University Medical Center for animal care and support during the operations.

\section{References}

1. Graham TP Jr. Ventricular performance in congenital heart disease. Circulation. 1991;84:2259-74.

2. Redington AN, Rigby ML, Oldershaw P, Gibson DG, Shinebourne EA. Right ventricular function 10 years after the Mustard operation for transposition of the great arteries: analysis of size, shape, and wall motion. Br Heart J. 1989;62:455-61.

3. Chow E, Farrar DJ. Effects of left ventricular pressure reductions on right ventricular systolic performance. Am J Physiol Heart Circ Physiol. 1989;257(suppl):H1878-85.

4. Santamore WP, Dell'Italia LJ. Ventricular interdependence: significant left ventricular contributions to right ventricular systolic function. Prog Cardiovasc Dis. 1998;40:289-308.

5. Park CH, Nishimura K, Kitano M, Matsuda K, Okamoto Y, Ban T. Analysis of right ventricular function during bypass of the left side of the heart by afterload alterations in both normal and failing hearts. J Thorac Cardiovasc Surg. 1996;111:1092-102.

6. Farrar DJ, Compton PG, Dajee H, Fonger JD, Hill JD. Right heart function during left heart assist and the effects of volume loading in a canine preparation. Circulation. 1984;70:708-16.

7. Bove AA, Santamore WP. Ventricular interdependence. Prog Cardiovasc Dis. 1981;23:365-88.

8. Leeuwenburgh BPJ, Helbing WA, Steendijk P, Schoof PH, Baan J. Biventricular systolic function in young lambs subject to chronic systemic right ventricular pressure overload. Am J Physiol Heart Circ Physiol. 2001;281:H2697-704.

9. Karunanithi MK, Michniewicz J, Young JA, Feneley MP. Effect of acutely increased left ventricular afterload on work output from the right ventricle in conscious dogs. J Thorac Cardiovasc Surg. 2001; 121:116-24.
10. Brookes C, Ravn H, White P, Moeldrup U, Oldershaw P, Redington A. Acute right ventricular dilatation in response to ischemia significantly impairs left ventricular systolic performance. Circulation. 1999;100:761-7.

11. de Vroomen M, Cardozo RH, Steendijk P, van Bel F, Baan J. Improved contractile performance of right ventricle in response to increased RV afterload in newborn lamb. Am J Physiol Heart Circ Physiol. 2000;278:H100-5.

12. Baan J, Van der Velde ET, de Bruin HG, Smeenk GJ, Koops J, van Dijk AD, et al. Continuous measurement of left ventricular volume in animals and humans by conductance catheter. Circulation. 1984;70: $812-23$.

13. Brookes CI, White PA, Bishop AJ, Oldershaw PJ, Redington AN, Moat NE. Validation of a new intraoperative technique to evaluate load-independent indices of right ventricular performance in patients undergoing cardiac operations. J Thorac Cardiovasc Surg. 1998;116: 468-76.

14. Derrick GP, Narang I, White PA, Kelleher A, Bush A, Penny DJ, et al. Failure of stroke volume augmentation during exercise and dobutamine stress is unrelated to load-independent indexes of right ventricular performance after the mustard operation. Circulation. 2000; 102(suppl 3):III154-9.

15. Kitano M, Nishimura K, Hee PC, Okamoto Y, Ban T. Right ventricular function evaluated by volumetric analysis during left heart bypass in a canine model of postischemic cardiac dysfunction. $J$ Thorac Cardiovasc Surg. 1995;109:796-803.

16. Suga H, Sagawa K. Instantaneous pressure-volume relationships and their ratio in the excised, supported canine left ventricle. Circ Res. 1974;35:117-26.

17. Little WC. The left ventricular $\mathrm{dP} / \mathrm{dtmax}$-end-diastolic volume relation in closed- chest dogs. Circ Res. 1985;56:808-15.

18. Glower DD, Spratt JA, Snow ND, Kabas JS, Davis JW, Olsen CO, et al. Linearity of the Frank-Starling relationship in the intact heart: the concept of preload recruitable stroke work. Circulation. 1985;71:9941009.

19. Dickstein ML, Yano O, Spotnitz HM, Burkhoff D. Assessment of right ventricular contractile state with the conductance catheter technique in the pig. Cardiovasc Res. 1995;29:820-6.

20. Karunanithi MK, Michniewicz J, Copeland SE, Feneley MP. Right ventricular preload recruitable stroke work, end-systolic pressurevolume, and $\mathrm{dP} / \mathrm{dtmax}$-end-diastolic volume relations compared as indexes of right ventricular contractile performance in conscious dogs. Circ Res. 1992;70:1169-79.

21. Little WC, Cheng CP, Mumma M, Igarashi Y, Vinten-Johansen J, Johnston WE. Comparison of measures of left ventricular contractile performance derived from pressure-volume loops in conscious dogs. Circulation. 1989;80:1378-87.

22. Leeuwenburgh BPJ, Steendijk P, Helbing WA, Baan J. Indexes of diastolic RV function: load dependence and changes after chronic RV pressure overload in lambs. Am J Physiol Heart Circ Physiol. 2002; 282:H1350-8.

23. Glantz SA, Slinker BK. Primer of applied regression and analysis of variance. New York: McGraw-Hill; 1990.

24. Fukamachi K, Asou T, Nakamura Y, Toshima Y, Oe M, Mitani A, et al. Effects of left heart bypass on right ventricular performance. Evaluation of the right ventricular end-systolic and end-diastolic pressure-volume relation in the in situ normal canine heart. $J$ Thorac Cardiovasc Surg. 1990;99:725-34.

25. Park CH, Nishimura K, Kitano M, Okamoto Y, Ban T. Right ventricular performance is impaired by full assist of left heart bypass. Analysis of right ventricular performance against change in afterload in heart failure models. ASAIO J. 1994;40:M303-8.

26. Miyamoto AT, Tanaka S, Matloff JM. Effects of left heart bypass on right ventricular function. Trans Am Soc Artif Intern Organs. 1982; 28:543-6.

27. Nishigaki K, Matsuda H, Hirose H, Nakano S, Ohtani M, Ohkubo N, et al. The effect of left ventricular bypass on the right ventricular function: experimental analysis of the effects of ischemic injuries to the right ventricular free wall and interventricular septum. Artif Organs. 1990;14:218-23. 
28. Yamaguchi S, Harasawa H, Li KS, Zhu D, Santamore WP. Comparative significance in systolic ventricular interaction. Cardiovasc Res. 1991;25:774-83.

29. King ME, Braun H, Goldblatt A, Liberthson R, Weyman AE. Interventricular septal configuration as a predictor of right ventricular systolic hypertension in children: a cross-sectional echocardiographic study. Circulation. 1983;68:68-75.

30. Moon MR, Castro LJ, DeAnda A, Tomizawa Y, Daughters GT, Ingels NB Jr, et al. Right ventricular dynamics during left ventricular assistance in closed- chest dogs. Ann Thorac Surg. 1993;56:54-66.

31. Markley JG, Nicolosi AC. Effects of left heart assist on geometry and function of the interventricular septum. Ann Thorac Surg. 1996;62: 1752-8.

32. Nelson GS, Sayed-Ahmed EY, Kroeker CA, Sun YH, Ter Keurs HE, Shrive NG, et al. Compression of interventricular septum during right ventricular pressure loading. Am J Physiol Heart Circ Physiol. 2001; 280:H2639-48.

33. Cochrane AD, Karl TR, Mee RB. Staged conversion to arterial switch for late failure of the systemic right ventricle. Ann Thorac Surg. 1993;56:854-61.

34. Bjarke BB, Kidd BS. Congenitally corrected transposition of the great arteries. A clinical study of 101 cases. Acta Paediatr Scand. 1976;65: 153-60.

35. Sano T, Riesenfeld T, Karl TR, Wilkinson JL. Intermediate-term outcome after intracardiac repair of associated cardiac defects in patients with atrioventricular and ventriculoarterial discordance. Circulation. 1995;92(suppl):II272-8.

36. Sidi D, Kuipers JR, Heymann MA, Rudolph AM. Recovery of cardiovascular function in newborn lambs after thoracotomy. Pediatr Res. 1982;16:705-10.

\section{Bound volumes available to subscribers}

Bound volumes of The Journal of Thoracic and Cardiovascular Surgery are available to subscribers (only) for the 2003 issues from the Publisher, at a cost of $\$ 134.00$ for domestic, $\$ 165.85$ for Canadian, and $\$ 155.00$ for international subscribers for Vol 125 (January-June) and Vol 126 (July-December). Shipping charges are included. Each bound volume contains a subject and author index and all advertising is removed. The binding is durable buckram with the Journal name, volume number, and year stamped in gold on the spine. Payment must accompany all orders. Contact Mosby, Subscription Customer Service, 6277 Sea Harbor Dr, Orlando, FL 32887, USA; phone 800-654-2452 or 407-345-4000.

Subscriptions must be in force to qualify. Bound volumes are not available in place of a regular Journal subscription. 